\title{
Mental Disorder and Suicide in Third Age Individuals
}

\author{
Olga Velentza ${ }^{1}$ \\ University of Athens
}

\author{
Evengelos Fradelos ${ }^{2}$ \\ State Mental Hospital
}

\begin{abstract}
In later life, suicide is at least two times more common than in the general population. Although the number of suicide attempts decreases in old age, the number of successful suicides increases. It is telling that while there is one fatality for every 10-20 suicide attempts in the general population, a fatal suicide occurs once in every 4 attempts in the elderly. It should be noted that suicidal behavior in the elderly indicates a genuine wish to die and is highly related with depression. The purpose of this study is to record the problems that lead older people to attempt suicide and to find ways to treat mental disorders in order to reduce suicide attempts. The results show that according to epidemiological studies, the overall prevalence of mental disorders in the elderly is approximately $25 \%$. A third of this percentage represents serious disturbances, $4-5 \%$ represent senile and arteriosclerotic dementia, 1\% represents melancholic situations, 1- 2\% represent chronic delusional states, and two thirds represent mild or minor disorders, mental retardation that has not reached dementia, depressive or established character neuroses. Senile psychiatric diseases occupy the third place among all illnesses after cardiovascular disease and cancer. According to current predictions, is expected to increase by $300 \%$ over the next 30 years. This study's conclusions state that both demographic and epidemiological studies foresee rising problem in the following years which will be exclusively related to the gerontology sector given that one in every two Europeans will be over 55 years of age. This group of physical, social, economic, political, and pathological problems will have a haunting effect on European society.
\end{abstract}

Keywords

Third age $\bullet$ Elderly suicidal behavior $\bullet$ Mental disorders

1 Correspondence to: Olga Velentza, Psychiatric Nurse R.N. Aiginiteio Hospital National and Kapodistrian University of Athens, Greece. Email:olyvelen@gmail.com

2 2nd Psychiatric Department, State Mental Hospital, Athens, Greece. Email: evagelosfradelos@hotmail.com

Citation: Velentza, O., \& Fradelos, E. (2016). Mental disorder and suicide in third age individuals. Sanitas Magisterium, 2, 111-119. http://dx.doi.org/10.12738/SM.2016.2.0020 
The "Third Age" has gained credence as a phase in human life with its own particular characteristics. Its features not only have to do with physical and/or physiological impairment or with the occurrence of either occasional or chronic diseases, but also with less frequent participation in activities and changes in one's social life and relationships with others. Older individuals are somehow expected to prove almost on a daily basis that they have not "begun to lose it" (in reference to their mental health), and that they can meditate and participate in daily life as they had done so in the past. Moreover, they are expected to prove that they still have wishes and goals (Soldatos \& Lykouras, 2000).

Factors that possibly affect etiopathogenetic the emergence of mental disorders in third age are:

\section{Factors pertaining to the individual}

- Pre-existing psychiatric disorders.

- Organic lesions on the brain.

- Frequent physical illnesses or chronic diseases.

- Chronic use of various drugs that can lead to psychiatric symptomatology.

- Resurgence of previous diseases.

\section{Factors pertaining to the social environment}

- In a society that gives importance to performance and consumerism, the weak and inefficient are less accepted.

- The role of the elderly is predefined, and the elderly must adjust to this role.

- Although retirement is generally better tolerated, losing one's partner often leads to depressive states.

- Living conditions sometimes leave an elderly individual no freedom of movement.

- Chronic loneliness can lead to psychiatric problems. In such cases, the admission to an institution can function therapeutically. Provided of course, that it will be an institution in which the elderly can partake in activities and communicate with peers.

- However, maintaining one's mental health and receiving physical care are not elderly individuals' sole concern, they also desire to participate in independent activities and creative occupations. As such, institutions are mostly seen as undesirable by elderly individuals. 
- In most cases, by becoming one of its many anonymous occupants, elderly individuals lose even the luxury of solitude when residing in institutions (Gournellis, 2011).

\section{The Psychopathology of Suicide in the Elderly}

The psychiatric disordered elderly person rarely seeks medical assistance by himself. Often their environment considers even symptoms of advanced psychiatric disease or incipient signs of an organic psychosyndrome as a normal aging phenomena. This then results in elderly patients receiving specialized medical assistance at fairly advanced situations. It is estimated that almost every third patient admitted to a psychiatric hospital is over 65 years of age.

Most authors agree that the frequency in which elderly individuals are admitted for diagnoses is as follows:

- Organic psychosyndrome.

- Depressive syndrome.

- Organic psychoses.

- Psycho reactive situations.

- Dependence diseases (Gulbinat, 1996).

\section{Classification of Psychiatric Disorders in Old Age}

1. The effect of age on existing psychiatric disorders.

$\checkmark$ Neurotic disorders. Such disorders emerge during the 2nd to 3rd decade of life and some forms during the 4th and 5th decades as a result of factors, events, or experiences encountered during the respective phases. Neurotic disorders, perhaps because of their relationship with releasing situational factors, usually decrease in intensity at older ages.

$\checkmark$ Personality disorders. Assuming that a relatively stable environment exists, they lose the intensity and dramatization of their characteristics.

$\checkmark$ Affective psychoses. With the passage of time, an individual displays a seemingly milder symptomatology, at least in regards to the intensity of the symptoms.

Although depressive phases are less dramatic, they last longer and are more resistant to medical treatment. Usually we observe a change of one's psychopathological picture, manifesting as "somatization" of symptomatology and "hypochondriacal" ideation. 
Depressive thoughts acquire increased paranoid content, especially in lonely individuals.

Suicidal acts increase in comparison to the average of other population groups.

A similar development is seen in bipolar disorder (manic depression). In old age, one exhibits stronger confusional symptoms and hyperactivity during the manic phase (Clark et al., 2011; Folks \& Ford, 1994; Soldatos \& Lykouras, 2000).

Psychotic schizophrenic disorders. Factors that appear to be associated with the final development of a schizophrenic disorder associated with:

$\checkmark$ The structure of one's personality.

$\checkmark$ One's social environment.

$\checkmark$ One's general physical health.

Particularly the latter two factors will determine to a great extent the clinical picture of the disease in the elderly.

2. Emerging psychiatric disorders in old age.

These include:

$\checkmark$ Organic psychosyndromes,

$\checkmark$ Depressive syndromes, and to a lesser extent:

$\checkmark$ Paranoid - hallucinatory states, and

$\checkmark$ Neurotic images with predominant symptoms the anxiety.

Organic psychosyndromes. These types of mental functional disorders are due to lesions of cerebral substance. The most common causes of organic psycho syndromes in old age are those that lead to the progressive degeneration of brain cells, either due to aging or to vascular disorders, metabolic diseases, or the chronic use of toxic substances. Most organic psychosyndromes in old age cover various types of dementia, with the main representatives being of the Alzheimer's type and senile dementia. This is due to a generalized cerebral atrophy which progresses over time and becomes apparent after the age of 65. The discount of mental abilities is gradual and progressive, without acute phases.

Depressive syndromes. The clinical picture of depression in advanced age has all the features of involutional melancholia, but at a heavier form. The loss of contact with 
persons, work etc. combined with marginalization, a lack of goals and purpose in life, being isolated from younger members of one's family and/or society, and a gradual reduction of physical capabilities all play a part in leading one to a depressive situation.

The reduction of activities and interests, increases the death anxiety which aggravates, any depressive situation. Depressive syndromes in the elderly may manifest in different forms, with one form manifesting as a primary disorder or with the picture of a major episode of depression, with all the characteristic symptoms of depressive syndrome (loss of interest, lack of will, incriminating thoughts and feeling of worthlessness, sleep disorders, psychomotor disorders, loss of appetite, etc). Depressive syndromes may also manifest as a chronic dysthymia with similar symptoms, albeit appearing significant lighter and thus more easily tolerated by the environment. In these cases the patient's environment considers such a situation to be "normal" for one's age and often does not care to seek medical help. When care is finally sought however, a long time has passed during which the elderly individual has lived without experiencing joy or pleasure.

Also frequent is the appearance of pseudoneurotic images as a hypochondriac behavior of various physical complaints or functional disorders, without the visible presence of depressive affect. In most of these cases consist of hidden depression characterized as informal (Christensen, Thomsen, Pedersen, Gram, \& KraghSorensen, 1985; Gournellis, 2011; Paraschos, 1996).

\section{Suicide in Third Age}

Statistics show that suicide has occurs at a higher frequency in old age than in other periods of life. For males, instances of suicide nearly double after 65 years of age and quadruple after 80 .

The risk of suicide increases in cases of:

- Physical or mental illness.

- Feelings of being abandoned.

Other risk elements that should be evaluated in order to predict suicide attempts:

- An irreversible depressed mood.

- The intense feeling of loneliness and of old age that seems to dominate one's average mood.

- Previous suicide attempts, even if the previous reasons (releasing causes) no longer practically exist. 
- Physical diseases that constitute potential risk to one's life or a chronic obsessive pathological state.

- A dependence on alcohol or other pharmaceutical formulations.

- Withdrawal of interest, even from past favorite activities (Katona \& Katona, 2000; Wiktorsson et al., 2013).

Since the elderly attempting such an act almost always wants to die, suicide attempts by the elderly are rarely impulsive and are most often well planned. The suicidal act is not an attention seeking act that often characterizes suicidal acts in younger age groups. Often an elderly individual planning his suicide is tired and sees death to be the expectation of release from the tortures of everyday life.

When observing a suicidal mood in an elderly person, two things should be taken into consideration in order to tackle it successfully:

(1) An elderly person who shows a general improvement in his/her psyche after a period of depression during which $\mathrm{s} /$ he contemplated suicide or not may indicate that $\mathrm{s} /$ he has reached his/her final decision. Such an improvement should not automatically be seen as a relief, but should lead to increased interest and care for the individual.

(2) Although many antidepressants provide a feeling of energy at first, users' antidepressant actions resume after several days. As a result, antidepressants can actually "push" an elderly person to attempt suicide by providing energy for it. For this reason, antidepressant products should be given with great care, under supervision, and with the combined administration of a sedative so as to prevent the emergence of energy until it is ensured that the antidepressant has taken effect (Patsakas, 1992; Small et al., 1997).

\section{Results}

According to the latest data from the US National Center for Health Statistics, suicide ranked as the ninth leading cause of death in the United States in 1992, being responsible for 30,484 deaths. In the general population, the suicide rate was 12.0 per 100,000 . Contrary to general believe, the rate was higher among the elderly (defined as people 65 years old and older), reaching 19.1. More distressing, this rate increased to 22.8 among people aged $75-\mathrm{b} 84$.

By comparison, while the elderly constituted $12.6 \%$ of the US population in 1992 , they corresponded to $20.2 \%$ of suicides. Those aged 15-24, however, accounted for $14.2 \%$ of suicides. A demographic analysis of these data reveals four critical importance variables affecting the rates of suicide in the elderly: gender, race, marital status, and the method of suicide attempted (Blazer, 2002). 
Of these variables, gender was found to be the more impressive, with successful attempts of suicide by men being four times more than those of women in 1992. Race, specifically the addition of Caucasians as demographic factor, further increased risk of attempting suicide. Although the suicide rate for white males increased progressively with increasing age, the percentages of Hispanics and of Afro-Americans were seen to peak at early maturity, followed by a decline falling thereafter.

The suicide rate in each family category, except for widowers was also observed to be higher in the elderly.

Finally, older people, both men and women, were more likely to use guns to commit suicide. In 1992, 68.7\% of elderly suicides were performed with guns, compared with $60 \%$ of all suicides that occurred in the United States. Men used firearms seven times more often than women, while white women outnumbered men in the use of solid or liquid poisons (Anstey \& Brodaty, 1995; Juurlink et al., 2004).

Although the overall suicide rate for the adult population in the United States has remained relatively stable since the Second World War, the suicide rate of elderly individuals decreased after the Great Financial Crisis in low percentage- record of $17.1 \%$ in 1981 . McIntosh et al. supposed that the decrease was due primarily to fewer suicides by men (especially white men) and the larger size of women as a group.

The effects of specific periods, such as times of increased political and social activism, economic security, and medical/psychiatric progress have been considered of great importance for this development. In contrast, the 1980 s was marked by an alarming $20 \%$ increase in elderly suicide rates, while the total number of suicides increased by $40 \%$ in the entire population.

Under assumptions proposed to explain the recent phenomenon, the increase in suicides recorded reflects an increase in the total elderly population and perhaps the improved collection and reporting of data. The most important factor, however, is the political and economic uncertainty of the last decade, as this may account for the increase, just as in the Great Depression during the 1930s (Baldwin \& Johnson,1995; Baumann \& Bertschy, 1993) .

\section{Discussion}

Incidents of suicide by elderly people are rising rapidly. Since 1980, the incidence of suicide in people over 65 has increased at a steady pace with experts predicting that if no action is taken, this phenomenon will continue.

Despite the fact that the overall rate of suicide in the elderly is less when compared to that observed in young people, the frequency of success in the elderly suicide 
attempts is greater. Social factors such as isolation, reduced mobility, psychological factors (loss of fitness and financial security), and biological factors (the disturbance of catecholamine neurotransmitter signals-especially norepinephrine and serotonin) appear to play an important role in increasing the incidence of suicide in the elderly. Of these factors, it should be noted that since biological factors play an important role in the metabolism of these neurotransmitters, they may cause an increase in depressive episodes in the elderly.

Depression is one of the major causes of suicide. Older men tend not to admit that they suffer from depression in comparison to women and therefore do not receive proper treatment.

The degree of despair and resignation from life and the rejection of any effort and/or prospect for future improvement combined with contemplation of suicide contemplation and guilt often leads one to decide to attempt suicide (Beaumont, 1989; Hyttel, 1994; Overø, 1984).

\section{Conclusions}

Demographic and epidemiological studies indicate an emerging pandemic in the following years related exclusively to the gerontology sector given that one in every two Europeans will be over 55 years of age. This group of physical, social, economic, political, and pathological problems will have a haunting effect on European society (Gottfries, Karlsson, \& Nyth, 1992).

\section{References}

Anstey, K., \& Brodaty, H. (1995). Antidepressants and the elderly: Double-blind trials 19871992. International Journal of Geriatric Psychiatry, 10(4), 265-279.

Baldwin, D., \& Johnson, F. N. (1995). Tolerability and safety of citalopram. Reviews in Contemporary Pharmacotherapy, 6, 315-325.

Baumann, P., \& Bertschy, G. (1993). Pharmacodynamic and pharmacokinetic interactions of Selective Serotonin Re-Uptake Inhibiting Antidepressants (SSRIs) with other psychotropic drugs. Nordic Journal of Psychiatry, 47(sup30), 13-19. http://dx.doi. org/10.3109/08039489309104120

Beaumont, G. (1989). Suicide and antidepressant over dosage in general practice. British Journal of Psychiatry, 155(Suppl. 16), 27-31.

Blazer, D. G. (2002). Epidemiology of depression: Prevalence and incidence. In J. R. M. Copeland, M. T. Abou-Saleh, \& D. G. Blazer (Eds.), Principles and practice of geriatric psychiatry (2nd ed., pp. 389-392). New York, NY: John Wiley \& Sons.

Christensen, P., Thomsen, H. Y., Pedersen, O. L., Gram, L. F., \& Kragh-Sørensen, P. (1985). Orthostatic side effects of clomipramine and citalopram during treatment for depression. Psychopharmacology, 86(4), 383-385. 
Clark, L., Dombrovski, A. Y., Siegle, G. J., Butters, M. A., Shollenberger, C. L., Sahakian, B. J., \& Szanto, K. (2011). Impairment in risk-sensitive decision-making in older suicide attempters with depression. Psychology and Aging, 26(2), 321.

Folks, D. G., \& Ford, C. V. (1994). Clinical features of depression and dysthymia. In J. R. M. Copeland, M. T. Abou-Saleh, \& D. G. Blazer (Eds.), Principles and practice of geriatric psychiatry (2nd ed., pp. 407-412). New York, NY: John Wiley \& Sons.

Gottfries, C. G., Karlsson, I. N. A. L., \& Nyth, A. L. (1992). Treatment of depression in elderly patients with and without dementia disorders. International Clinical Psychopharmacology, 6, 55-64.

Gournellis, P. (2011). Depression in the elderly: Modern data. Engephalos, 48, 141-142.

Gulbinat, W. H. (1996). The epidemiology of suicide in old age. Archives of Suicide Research, 2(1), 31-42. http://dx.doi.org/10.1080/13811119608258992

Hyttel, J. (1994). Pharmacological characterization of Selective Serotonin Reuptake Inhibitors (SSRIs). International Clinical Psychopharmacology, 9, 19-26.

Juurlink, D. N., Herrmann, N., Szalai, J. P., Kopp, A., \& Redelmeier, D. A. (2004). Medical illness and the risk of suicide in the elderly. Archives of Internal Medicine, 164(11), 11791184 .

Katona, C., \& Katona, P. (2000). Depression and anxiety attendant Sousse elderly. Athens: I. E. Vagionakis.

Overø, K. F. (1984). The role of pharmacokinetics in the development of new drugs: An illustration by studies on citalopram. Retrieved from https://bibliotek.dk/da/work/870970basis:06583105

Paraschos, I. (1996). Depression in late life. Greece: Lundbeck Hellas S.A.

Patsakas, I. (1992). The third age in the late 20th century. Athens: Pitsilas Publications.

Small, G. W., Rabins, P. V., Barry, P. P., Buckholtz, N. S., DeKosky, S. T., Ferris, S. H., ... \& McRae, T. D. (1997). Diagnosis and treatment of Alzheimer disease and related disorders: Consensus statement of the American Association for Geriatric Psychiatry, the Alzheimer's Association, and the American Geriatrics Society. Jama, 278(16), 1363-1371.

Soldatos, K., \& Lykouras, L. (2007). Textbook psychiatry Vol II Tomes. Athens, Greece: Publication Beta.

Wiktorsson, S., Berg, A. I., Billstedt, E., Duberstein, P. R., Marlow, T., Skoog, I., \& Waern, M. (2013). Neuroticism and extroversion in suicide attempters aged 75 and above and a general population comparison group. Aging \& Mental Health, 17(4), 479-488. 
